\title{
La pratique de l'irlandais et la minorité irlandophone
}

L'irlandais comme langue nationale

The practice of Irish and the Irish-speaking minority

\section{Tadhg Ó Hifearnáin}

\section{OpenEdition}

\section{Journals}

Édition électronique

URL : https://journals.openedition.org/lbl/1052

DOI : $10.4000 / \mathrm{lbl} .1052$

ISSN : 2727-9383

\section{Éditeur}

Université de Bretagne Occidentale - UBO

Édition imprimée

Date de publication : 1 juin 2015

Pagination : 81-97

ISBN : 979-10-92331-16-5

ISSN : 1270-2412

\section{Référence électronique}

Tadhg Ó Hifearnáin, «La pratique de l'irlandais et la minorité irlandophone », La Bretagne Linguistique [En ligne], 19 | 2015, mis en ligne le 01 mai 2021, consulté le 22 mai 2021. URL : http:// journals.openedition.org//bl/1052 ; DOI : https://doi.org/10.4000/lbl.1052

La Bretagne Linguistique est mise à disposition selon les termes de la Licence Creative Commons Attribution 4.0 International. 


\section{Tadhg Ó HIFEARNÁIN*}

\section{La pratique de l'irlandais et la minorité irlandophone}

\section{L'irlandais comme langue nationale}

\section{$\mathrm{L}$}

a situation actuelle de l'irlandais présente de nombreux parallèles avec d'autres langues minoritaires en Europe et dans le monde, surtout là où les États ou les pouvoirs publics ont essayé d'intervenir dans les situations de langues en contact pour produire un résultat social différent de ce que la dynamique déjà en place aurait assuré sans une telle intervention. Cependant, dans son étude de l'interaction de la politique de la langue et de sa reproduction sociale en Irlande, Pádraig Ó Riagáin ${ }^{1}$ constate que le pays se distingue de la plupart de ces autres États et territoires à deux égards. Tout d'abord, la politique de la langue irlandaise est en opération pour une période beaucoup plus longue que dans ces autres territoires, ce qui rend l'expérience du pays utile pour tester les effets à long terme de l'interface Étatcommunauté dans la gestion des langues, notamment à l'égard de la politique scolaire. Deuxièmement, il observe que, contrairement à d'autres situations où on essaie de protéger une minorité linguistique, en Irlande l'État a tenté de faire face à son problème de langue mino-

* Université de Limerick, Irlande.

1. Pádraig Ó Riagáin, Language Policy and Social Reproduction: Ireland 18931993, Oxford, Clarendon Press, 1997. 
ritaire en cherchant à la rétablir en tant que langue nationale : «Aucun autre problème de langue minoritaire en Europe n'a été abordé ainsi, bien que les cas un peu particuliers (mais relativement récents) des langues régionales en Espagne aient quelques similitudes ${ }^{2}$.»

En revanche, on peut constater que l'idéologie qui mène à faire d'une langue minoritaire une langue à vocation nationale est bien répandue chez les militants et mouvances en faveur des langues minoritaires en Europe. Si les cas du catalan, du galicien et du basque ressemblent un peu au cas irlandais, c'est-à-dire que les autorités publiques veulent faire d'une langue autochtone minorisée la langue propre d'une région, voire d'un État autonome, bien d'autres militants aimeraient bien en faire autant s'ils pouvaient s'offrir les moyens de le faire. L'idée qu'une langue minoritaire appartient non seulement à ses locuteurs et à leurs descendants mais aussi au territoire et donc à tout ces habitants contemporains, peu importe leurs origines, semble bien ancrée dans l'idéologie des militants des langues minoritaires en Europe. Ce n'est pas l'action réelle de la promotion de la langue indigène minorisée au niveau de «première langue officielle» par l'État nouvellement indépendant qui rend le cas irlandais instructif pour ce qui concerne les cas des politiques linguistiques nationales et régionales envers les langues minoritaires, mais cette idéologie linguistique qui a amené l'État à mener cette action. Si la langue minoritaire est devenue langue nationale, il est implicite que la minorité de la population qui a toujours utilisé l'irlandais comme langue maternelle et langue du foyer ne pouvait plus être considérée par l'État comme étant un groupe culturel distinct du reste de la population. Les irlandophones ne seraient donc pas une «minorité», mais simplement des gens comme les autres, mais des gens qui avaient quand même plus de compétences dans la langue nationale. La majorité anglophone irlandaise est, selon cette idéologie, une population qui a changé de pratique langagière, mais à qui appartient toujours l'irlandais. Il n'y a pas de minorités linguistiques en Irlande, croit-on, mais il existe plutôt divers degrés de capacités linguistiques et diverses pratiques langagières en ce qui concerne l'irlandais.

Cette idéologie fondatrice de la politique linguistique en Irlande nourrit toujours le discours officiel envers l'irlandais, mais une

2. Op. cit. p. vii. 
partie de la population anglophone n'a peut-être jamais souscrit à cet engagement et il est évident que ces gens trouvent à nouveau leur voix dans le cadre de la démocratisation partielle de la planification linguistique dans les régions de la Gaeltacht. De même, une bonne partie de la population irlandophone se voit toujours comme une minorité culturelle en Irlande ${ }^{3}$.

\section{Le nombre de locuteurs de l'irlandais}

Les enquêtes montrent régulièrement que la majorité des personnes nées en République irlandaise connaissent l'irlandais, au moins un peu, grâce à son enseignement obligatoire tout au long de leur scolarité, ce qui est un résultat de la politique désignant l'irlandais comme langue nationale. Mac Gréil et Rhatigan ${ }^{4}$, sur la base d'une enquête nationale de 1015 adultes, montrent, par exemple, que seulement $16 \%$ de la population née en Irlande ne parlent pas du tout l'irlandais. La grande majorité, environ $80 \%$, des Irlandais, soutient l'enseignement obligatoire de l'irlandais à l'école, et la politique nationale en faveur de la langue (subventions socio-économiques pour la Gaeltacht, émissions de télévision et radio, signalisation en irlandais, etc.), du moins depuis que l'on a commencé de les sonder régulièrement dans les années soixante-dix ${ }^{5}$. La compétence passive dans la langue se révèle, par exemple, par le fait que le quart de la population regarde les émissions de télévision en irlandais (quoique sous-titrés en anglais) de temps à autre. Même si la politique en faveur de la langue a donc connu une certaine réussite, très peu d'Irlandais la parlent couramment et régulièrement, et peu se renseignent sur les

3. Tadhg Ó HIFEARNÁIN, «Paradoxes of engagement with Irish language community management, practice and ideology», in P. Austin, J. Sallabank (dir.), Endangered Languages. Beliefs and ideologies in Language Documentation and Revitalization, Oxford, Oxford University Press, 2014, p. 29-51. Simon WARREN, «The making of Irish-speaking Ireland: the cultural politics of belonging, diversity and power», Ethnicities, 2012, 12, p. 317-334.

4. Micheál MAC GréIL, Fergal Rhatigan, The Irish Language and the Irish People: Report on the Attitudes Towards, Competence in and Use of the Irish Language in the Republic of Ireland 2007-08, Maynooth, Department of Sociology, National University of Ireland, Maynooth, 2009.

5. Padraig Ó RiagÁin, Mícheál Ó GLIASÁIn, National Survey on Languages 1993 : Preliminary Report, 1994, Dublin, Institiúid Teangeolaíochta Éireann. 
détails de cette politique linguistique, de ses réussites et ses échecs, parce qu'elle ne touche pas beaucoup leur vie quotidienne en dehors du système scolaire.

D'après le recensement de 2011 il y a 1774437 locuteurs, soit $40,6 \%$ de la population de la république. Le nombre de locuteurs est en progression depuis la création de l'État (sauf 1946), même si le pourcentage de locuteurs dans la population a plafonné autours de $41 \%$ depuis 1996 (voir tableau 1), suite en partie à une forte immigration depuis les années 2000 . Actuellement $12 \%$ de la population n'est pas de nationalité irlandaise, le plus grand groupe d'immigrés étant les Polonais (4\% de la population), qui devancent les Britanniques et puis les Lituaniens, les Lettons et divers autres nationalités d'Europe, d'Afrique, d'Asie et des Amériques. Comme ces nouveaux venus n'ont pas connu l'école en Irlande, la grande majorité des immigrés n'a pas appris la langue comme l'a fait la grande majorité des citoyens.

Tableau 1. Locuteurs d'irlandais (seulement pour la République irlandaise, 3 ans d'âge et plus) selon les recensements de 1926 à $2011^{6}$

\begin{tabular}{|c|c|c|c|}
\cline { 2 - 4 } \multicolumn{1}{c|}{} & Population de l'État & Parlent l'irlandais & Pourcentage \\
\hline 1926 & 2802452 & 540802 & $19,3 \%$ \\
\hline 1936 & 2806925 & 666601 & $23,7 \%$ \\
\hline 1946 & 2771657 & 588725 & $21,2 \%$ \\
\hline 1961 & 2635818 & 716420 & $27,2 \%$ \\
\hline 1971 & 2787448 & 789429 & $28,3 \%$ \\
\hline 1981 & 3226467 & 1018413 & $31,6 \%$ \\
\hline 1986 & 3353632 & 1042701 & $31,1 \%$ \\
\hline 1991 & 3367006 & 1095830 & $32,5 \%$ \\
\hline 1996 & 3479648 & 1430205 & $41,1 \%$ \\
\hline 2002 & 3750995 & 1570894 & $41,9 \%$ \\
\hline 2006 & 3956964 & 1656790 & $41,9 \%$ \\
\hline 2011 & 4370631 & 1774437 & $40,6 \%$ \\
\hline
\end{tabular}

6. Central Statistics Office, Census 2006. Volume 9 - Irish Language, 2007, Dublin, Stationary Office, p. 11.

Central Statistics Office, This is Ireland: Highlights from Census 2011, Part 1, Dublin, Stationery Office, p. 98. 
Cependant, nous ne savons pas exactement ce que les gens entendent quand ils se proclament capable de parler la langue en réponse au recensement. Pourquoi, par exemple, le pourcentage de «locuteurs » recueilli par le recensement ne touche-t-il pas les quelques $85 \%$ de la population qui ont appris l'irlandais à l'école ? En fait, le recensement pose la question mais ne définit pas ce que «parler» veut dire. Plusieurs enquêtes et sondages effectués au plan national depuis les année soixante-dix, dont Mac Gréil et Rhatigan en 20072008 , montrent que la population répond d'une manière plus positive quand on lui donne une série de choix de compétences linguistiques au lieu d'un simple « oui » ou « non » (voir tableau 2$)^{7}$.

Tableau 2. Autoévaluation de compétences en irlandais 2007-2008 (répondants nés en Irlande)

\begin{tabular}{|l|r|}
\hline Bien couramment & $9,1 \%$ \\
\hline Moyennement & $22,1 \%$ \\
\hline Pas couramment & $15,9 \%$ \\
\hline Un peu & $36,9 \%$ \\
\hline Pas du tout & $16,0 \%$ \\
\hline
\end{tabular}

Ce chiffre de $9,1 \%$ de la population nous donnerait quelque 393000 locuteurs compétents, un chiffre qui semble toujours un peu élevé et en décalage avec le nombre de locuteurs qui utilisent la langue au quotidien. Encore est-il que l'autoévaluation ne donne pas de chiffres fiables aux linguistes, mais plutôt des indications sur le sentiment dans la population envers une langue qu'ils ont appris mais qu'ils ne pratiquent pas souvent.

Lors du recensement de 2011, il a été demandé à la population de décrire la fréquence avec laquelle elle parle l'irlandais et aussi si ces locuteurs au quotidien ou hebdomadaires le parlaient en dehors de l'école (pour éviter d'inclure les écoliers et leurs maîtres qui ne l'utilisent qu'à l'école, où la langue est obligatoire). Seulement 77185 personnes ont répondu qu'ils parlent l'irlandais quotidiennement en dehors du système éducatif, soit 1,8\% de la population. Encore est-il impossible de savoir exactement ce que les

7. Micheál MaC GrÉIL, Fergal Rhatigan, op. cit. p. 39-40. 
gens ont mis sous le terme de «parler quotidiennement», mais nos enquêtes auprès des locuteurs dans la Gaeltacht nous mènent à croire que ce groupe de la population nous fournit la base de la communauté linguistique, auquel il faut ajouter un certain nombre de gens qui parlent bien l'irlandais mais qui ne le font pas régulièrement parce qu'ils ne font pas partie de ce petit peuple de locuteurs quotidiens.

Un quart de ces locuteurs au quotidien ont moins de 18 ans, et la démographie (âge, profil social) des locuteurs réguliers correspond à peu près au profil de la population générale sauf quant au sexe. 42157 sont des femmes et 35028 des hommes $(54,6 \%$ et $45,4 \%$ du total), et les femmes sont plus nombreuses que les hommes dans toutes les catégories d'âge de moins de 54 ans. Ceci est remarquable car il s'agit d'une inversion des faits que l'on a connus au XIX et début du $\mathrm{XX}^{\mathrm{e}}$ siècle quand les femmes ont mené le changement linguistique antérieur dans les régions irlandophones, les hommes étant, comme cela est le modèle courant en sociolinguistique, plus conservateurs quant à la conservation de la langue locale. Nous voyons toujours ce profil dans les âges de 54 ans et plus, où les hommes irlandophones sont toujours plus nombreux que les femmes, mais les femmes sont plus nombreuses dans la population générale. Les femmes devancent les hommes aussi quand nous regardons les chiffres globaux de compétences que nous donne le recensement. 44,9\% des femmes s'autoévaluent comme locuteurs de l'irlandais contre 37,9\% des hommes. Il est probable que ces chiffres nous montrent un effet scolaire, surtout en dehors de la Gaeltacht. Les filles réussissent mieux à l'école et surtout dans les langues. La majorité des enseignants en primaire et les professeurs de langues dans le secondaire (collège et lycée) sont des femmes.

En fait, 3249 des locuteurs quotidiens sont eux-mêmes des instituteurs et 2074 sont des professeurs de collège/lycée ${ }^{8}$. Il est probable que ces gens sont des professeurs parce que c'est un métier prisé des irlandophones, et non parce que les maîtres d'école se mettent plus à l'irlandais dans leurs vies personnelles. Dans une grande enquête sociolinguistique de terrain dans la région Gaeltacht de Múscraí dans le Cork de 2000 à 2004, par exemple, nous avons rencontré beau-

8. Central Statistics Office, Profile 9 - What We Know, Dublin, Stationery Office, p. 30, p. 72. 
coup d'enseignants originaires de ce petit peuple irlandophone qui vivaient bien chez eux, mais qui étaient en poste dans les régions et villes qui les entourent. L'État a bien favorisé le recrutement d'enseignants d'irlandais chez les habitants de la Gaeltacht, notamment de 1927 à 1960 avec la création des Coláistí Ullmhúcháin «collèges préparatoires». Ils ont été conçus comme pensionnats d'éducation secondaire (dès l'âge de douze ans) de langue irlandaise dont les élèves devaient être admis dans les écoles normales pour devenir instituteurs et ainsi être nommés partout dans le pays. Le ministère de l'Éducation a décidé de créer ces écoles spéciales en 1926, et les trois premières des sept écoles qui devaient se créer (trois pensionnats pour les garçons catholiques, trois pour les filles catholiques et une école mixte pour les protestants) ont été ouvertes en 1927, les autres ayant été ouvertes avant 1930. Kelly ${ }^{9}$ indique que ces écoles n'ont pris qu'environ 25 élèves chacune au départ, mais quelques années après, elles avaient entre 550 et 600 élèves inscrits. On n'a pas admis de nouveaux étudiants dans ces pensionnats de 1939 jusqu'en 1942, parce que le nombre d'instituteurs qualifiés était déjà plus que suffisant. C'est durant cette période que le sort des collèges a été scellé, lorsque la nécessité d'avoir des collèges spéciaux de cette nature a été contestée étant donné le succès apparent des écoles ordinaires en fournissant des candidats de qualité pour les écoles normales. Quand les coláistí ullmhúcháin ont rouvert leurs portes en 1942, elles ne se sont que lentement remplies, avant que le ministre de l'Éducation annonce, en 1958, que l'accès à la formation des instituteurs se ferait désormais seulement par voie de résultats scolaires et entretien et donc qu'il n'y aurait plus de places garanties pour les élèves de ces pensionnats de la Gaeltacht. Néanmoins le métier d'enseignant est bien vu et prisé par beaucoup de gens de la Gaeltacht et le pourcentage de professeurs d'école parmi les gens en activité demeure relativement élevé dans ces régions. Par contre, étant donné l'image populaire des locuteurs d'irlandais comme paysans et pêcheurs, il est intéressant de noter qu'il n'y a que quelque 1033 agriculteurs parmi les locuteurs quotidiens.

9. Adrian Kelly, Compulsory Irish. Language and Education in Ireland 1870s-1970s, Dublin, Irish Academic Press, 2002. 
Les régions définies comme ayant une population irlandophone, la Gaeltacht, comptaient 96628 personnes en avril 2011, dont $69 \%$ se disaient irlandophones. Seulement 23175 de ces gens confirment qu'ils parlent l'irlandais quotidiennement. C'est-à-dire que seulement le tiers des locuteurs quotidiens habitent dans ces régions où une majorité de la population peut parler la langue, et les deux tiers des locuteurs quotidiens sont en dehors de la Gaeltacht.

En Irlande du Nord, on acquiert les données linguistiques sur la base d'autres questions dans le recensement. Lors du recensement de 2011, il a été demandé aux gens de dire s'ils se sentaient capables de comprendre, parler, lire et/ou écrire l'irlandais (et/ou l'Ulster Scots) et aussi quelle était leur «langue principale». 184898 ont répandu qu'ils avaient au moins une des compétences décrites en irlandais ( $10,65 \%$ de la population). Le pourcentage avoisine les $20 \%$ dans les régions à majorité catholique, où les gens n'ont pas seulement un sentiment plus proche du comportement culturel de leurs voisins du sud, mais où la langue est enseignée dans les écoles publiques catholiques - elle demeure absente dans les écoles publiques dites «d'État» et protestantes. Nous apprenons en outre que l'irlandais est en quatrième place dans la liste des « langues principales » en Irlande du Nord, après l'anglais (97\%), le polonais et le lituanien, avec 4164 locuteurs $(0,24 \%$ de la population).

On ne peut pas être totalement précis, mais il semble donc que nous ayons un noyau de 81349 locuteurs qui parlent l'irlandais quotidiennement, dont un peu plus du quart habitent dans la Gaeltacht et $5 \%$ en Irlande du Nord. Mais il faut se poser quelques questions sur la nature de cette population qui pratique l'irlandais, une population qui est entourée d'une plus grosse population à diverses compétences linguistiques et qui dépend de la volonté de la population générale de soutenir les services, les écoles, les médias, l'édition, etc. en irlandais. À quel point la minorité irlandophone est-elle une communauté à part, une bounded community, et comment peut-on devenir irlandophone aux yeux et aux oreilles des irlandophones de naissance ou de choix ? Pour essayer de répondre à ces questions, il faut d'abord considérer à quel point l'État admet par ses actions qu'il existe en réalité une minorité linguistique au sein de la population et si les locuteurs de l'irlandais eux-mêmes se considèrent comme une minorité linguistique et culturellement différente de la majorité anglophone. 


\section{La Gaeltacht et les irlandophones comme minorité linguis- tique}

L'État irlandais a donc choisi de protéger et de promouvoir sa langue minorisée comme partie intégrante de la politique nationale dès la fondation de l'État indépendant (de 1916 à 1922), en faisant de l'irlandais la seule langue «nationale ${ }^{10}$ et par conséquent première langue officielle d'État, à côté de l'anglais, langue majoritaire mais placée au deuxième rang par la Constitution (actuelle) de $1937^{11}$. Dans les années 1920, à l'instar des pratiques européennes de l'époque, notamment la gestion des minorités prévue dans les traités de paix de Paris et garantie par la Société des Nations, telles la protection de la population suédophone des îles Åland en Finlande, ou des droits linguistiques des germanophones de la Haute-Silésie polonaise, l'État a voulu définir le peuple des locuteurs natifs de l'irlandais de manière territoriale, tout en refusant d'admettre l'existence d'une minorité linguistique, l'irlandais étant la langue propre de tous les Irlandais selon le discours national. La délimitation de la Gaeltacht officielle s'est concrétisée progressivement de 1926 (Rapport de la Commission sur la Gaeltacht) ${ }^{12}$ à 1956 (création du ministère de la Gaeltacht, et la définition légale de son aire de travail), avec quelques petites additions en 1964, 1967 et $1982^{13}$.

Cette définition de la Gaeltacht comme territoire est assez nouvelle dans l'histoire culturelle de la langue. Dans le passé, le mot «Gaeltacht» signifiait le peuple de langue gaélique et non son territoire. Le dictionnaire irlandais-anglais du Père Patrick Dinneen, Foclóir Gaedhilge agus Béarla, publié en 1904 avant la création de l'État indépendant, et qui faisait référence jusqu'à la publication du Foclóir Gaeilge-Béarla de Niall Ó Dónaill en 1977, la définit ainsi :

Gaedhealtacht, -a, f., the state of being Irish or Scotch; Gaeldom, Irishry, the native race of Ireland; bean de'n ghaedheal-

10. Article 5 de la Constitution de l'État libre irlandais de 1922, Bunreacht Shaorstát Éireann.

11. Article 8.1 et 8.2 de la Constitution d'Irlande de 1937, Bunreacht na hÉireann.

12. Coimisiún na Gaeltachta [Gaeltacht Commission], Report, Dublin, Stationary Office, 1926.

13. Tadhg Ó HIfEARNÁIN, "Irish-speaking society and the state», in M. Ball, N. Müller (dir.), The Celtic Languages (2e édition), 2009, Londres et New York, Routledge, p. 539-586. (Surtout p. 557-564 pour la délimitation de la Gaeltacht.) 
tacht, a woman of the Irishry (Art McC.); g. Alban, the Highlands of Scotland.

Donc, la «Gaeltacht» VOULAIT DIRE «l'état d'être irlandais ou écossais», «appartenant au peuple irlandophone», ou décrivait un indigène d'Irlande ou des Hautes Terres écossaises (ou de l'île de Man). On le comprend toujours dans ce sens au sein du peuple (ou peuples) de langue gaélique en Irlande et en Écosse, mais pour la plupart, du moins en Irlande, c'est la définition de la Gaeltacht comme territoire officiel de langue irlandaise qui est la plus répandue. Cependant, nous avons déjà vu que la majorité des locuteurs habituels n'habite pas le territoire officiel de la dénommée Gaeltacht et plusieurs sociolinguistes ont demandé depuis longtemps comment on peut définir une communauté linguistique seulement par l'endroit où une partie de ses locuteurs habite, surtout dans le contexte moderne de migrations, de trajets quotidiens pour le travail, l'éducation, le commerce et la vie sociale, sans parler des réseaux sociaux électroniques.

De 2002 à 2012 l'État a entrepris une série de projets de réflexion sur l'avenir de la Gaeltacht, culminant dans la 20 Year Strategy for the Irish Language 2010-2030 et Acht na Gaeltachta (loi sur la Gaeltacht du 19 juillet 2012). La loi sur la Gaeltacht de 2012 prévoit d'établir un processus officiel de gestion sociolinguistique en divisant la Gaeltacht en "régions de planification linguistique» créées selon le pourcentage de locuteurs de l'irlandais et la dynamique de changement linguistique présent. Cette loi se veut inspirée des meilleures pratiques européennes dans la protection des langues minoritaires, mais ne réussit pas pour autant à s'écarter de deux éléments fondamentaux du discours national sur la langue: d'un coté, il n'existe pas de «minorité irlandophone» et de l'autre, la Gaeltacht et son peuple doivent se définir de manière territoriale. C'est vrai que la loi de 2012 prévoit la possibilité d'accorder un statut spécial de Gaeltacht aux réseaux irlandophones en dehors de la Gaeltacht officielle, mais ces réseaux doivent se trouver dans un endroit bien défini, et ni les critères pour permettre à un réseau de demander ce statut, ni ce que le statut d'«aire de planification linguistique en réseau» voudrait dire n'ont suscité d'explication de la part du gouvernement à ce jour. 
Le repli sur ce discours conservateur de territoire et de langue nationale est au cœur du rapport Comprehensive Linguistic Study of the Use of Irish in the Gaeltacht: Principal Findings and Recommendations ${ }^{14}$ de 2007, que le gouvernement a commandé en 2004 et qui a, en quelque sorte, établi les paramètres du débat public et gouvernemental sur la politique linguistique qui a mené à la nouvelle loi sur la Gaeltacht en 2012. Le Comprehensive Linguistic Study a utilisé les résultats du recensement de 2002 et de Scéim Labhairt na Gaeilge (une petite bourse octroyée aux familles irlandophones dans la Gaeltacht) pour délimiter trois zones de vitalité linguistique : la catégorie $A$ où la majorité ( $67 \%$ et plus) parle l'irlandais au quotidien, la catégorie $\mathrm{C}$ où la minorité la parle au quotidien (moins de $44 \%$ ), et quelques petits endroits en catégorie B qui sont entre les deux.

Ces zones ont été définies selon le cadre des «divisions électorales », l'unité géographique d'analyse statistique la plus petite du service national des statistiques (an Phriomhoifig Staidrimh/ Central Statistics Office). On a déjà mis ces divisions électorales en cause en 1956 quand la Gaeltacht actuelle a vu le jour car elles ne correspondent que rarement à ce que le peuple comprend lui-même de sa communauté, n'étant ni paroisse, ni secteur scolaire, ni autre unité culturelle ou socio-économique reconnue par les gens. On note, par exemple, qu'un des endroits les plus irlandophones du Donegal, Rann na Feirste, est placé dans la catégorie B (quand il aurait dû être bel et bien en catégorie $\mathrm{A}$ ) parce qu'il se trouve à quelques kilomètres du bourg largement anglophone de Anagaire, mais dans la même division électorale que ce centre de population avec lequel les gens de Rann na Feirste n'ont que peu de contacts, utilisant les écoles et les commerces de la grande paroisse irlandophone du Gaoth Dobhair voisin. On constate aussi que très peu de secteurs en catégorie A ont leurs propres écoles secondaires, magasins, terrains de sport, centres économiques ou de loisirs, qui sont partagés avec des divisions électorales d'à coté ou dans les petites villes et bourgs de catégorie C.

14. Conchúr Ó Giollagáin, Seosamh Mac Donnacha, Fiona Ní Chualáin, Aoife Ní ShÉAghdHA, and Mary O'Brien, Comprehensive Linguistic Study of the Use of Irish in the Gaeltacht: Principal Findings and Recommendations, 2007, Dublin, Stationery Office. 
La Comprehensive Linguistic Study contient les résultats de plusieurs volets importants de recherches, notamment sur le comportement linguistique des adolescents et sur le nombre de permis de construire des maisons de vacances dans les régions Gaeltacht $\mathrm{du}$ sud-ouest. Ses conclusions alimentent toutes son constat que l'irlandais est en déclin, et qu'il faut intervenir si l'on veut éviter la fin de son utilisation comme langue populaire de la Gaeltacht. Le rapport prend ainsi sa place dans une longue série de commentaires qui prédisent la fin de la langue depuis plusieurs siècles, faisant partie du «discours du menacé» ${ }^{15}$. Cependant, les liens instrumentaux entre les analyses statistiques, les résultats des projets de terrain et les recommandations du rapport en ce qui concerne la planification linguistique ne sont pas toujours évidents, et on constate que la plupart de ces recommandations pratiques ressemblent à celles du rapport du Commission de la Gaeltacht en 1926, qu'il s'agisse de la division de la Gaeltacht en zones selon le pourcentage de locuteurs, des questions scolaires, de commerce, des villes limitrophes et autres centres de population, du besoin d'obliger les parents à parler en irlandais avec leurs enfants, et bien d'autres. Il appelle au renouvellement des structures qui sont largement déjà en place, mais qui n'ont connu qu'un succès mitigé.

Cependant, on perçoit quelques différences d'esprit entre les rapports de 1926 et de 2007, même si l'idéologie de base reste similaire. Le rapport de 1926 présentait la Gaeltacht comme partie intégrante de la planification nationale de revitalisation de l'irlandais. On y a défini trois zones linguistiques dans le pays entier ; la FíorGhaeltacht («vraie Gaeltacht») avec $80 \%$ de locuteurs, la BreacGhaeltacht (Gaeltacht bigarrée ou rayée) où il y avait entre $25 \%$ et $79 \%$ de locuteurs, et puis le reste du pays. Le but de cette politique idéaliste, voire utopiste, était le même pour les trois zones : (re-) devenir irlandophone, et visait notamment l'irlandisation du système scolaire et de la fonction publique. Le rapport de 1926 n'envisageait donc pas la création d'une frontière géographique fixe entre les deux catégories de Gaeltacht et entre celles-là et le reste du pays car on la voulait dynamique, partie intégrante de la politique linguistique

15. Alexandre DuchêNe, Monica Heller (dir.), Discourses of Endangerment: Interest and Ideology in the Defense of Languages, 2007, Londres, Continuum. 
nationale en faveur de l'irlandais. On espérait que la BreacGhaeltacht deviendrait bientôt comme la Fíor-Ghaeltacht et que le reste du pays ressemblerait rapidement à la Breac-Ghaeltacht, avant de devenir vraiment irlandophone quand la jeune génération ayant fait ses études en irlandais serait grande. Le rapport de 2007 ne vise en rien l'extension de la Gaeltacht ni la gaélicisation du pays entier, mais parle de sa gestion comme région irlandophone, ou région à forte population irlandophone, en bref, où il fallait créer des aires de planification linguistique à buts différents taillés sur mesure pour les zones de catégorie A, B ou C. Il s'abstient, pour autant, d'identifier une minorité linguistique en décrivant chaque collectivité locale comme étant une seule population locale qui manifeste une compétence linguistique et une fréquence d'utilisation qui s'élèvent à tel ou tel pourcentage. Cette position place les recommandations $\mathrm{du}$ rapport dans une situation paradoxale. On admet le besoin de gérer différemment une population qui se distingue de la population nationale quant à ses pratiques linguistiques, mais on croit toujours dans l'idéologie linguistique fondatrice qui ne voit pas de différences culturelles entre les gens de la Gaeltacht elle-même, peu importe où ils se trouvent entre les deux points extrêmes de l'échelle sociolinguistique, entre les locuteurs habituels de l'irlandais et les anglophones monolingues dans ces mêmes paroisses.

\section{Une minorité culturelle de langue irlandaise ?}

Pour tenter d'engager les populations de la Gaeltacht dans l'aménagement linguistique, Údarás na Gaeltachta ${ }^{16}$ et le ministère responsable de la Gaeltacht ont entrepris une enquête standardisée entre 2007 et 2008 dans 28 des communautés qui n'avaient pas à ce moment préparé leur plan local. Les informations ont été recueillies par des groupes bénévoles communautaires qui ont interrogé 12271 participants. Les communautés concernées ne sont pas représentatives de la Gaeltacht entière, mais la fourchette est grande et comprend à la fois des régions fortes de «locuteurs quotidiens de l'irlandais» comme des communautés du sud du Connemara et des îles Árainn mais aussi des paroisses moins irlandophones de

16. L'agence locale pour le développement socio-économique de la Gaeltacht. 
la Gaeltacht officielle. Les résultats de ce projet, nommé Gaeilge 2010, montrent des différences marquées entre les locuteurs natifs et les locuteurs qui ne parlent pas couramment l'irlandais ou qui ne le parlent pas du tout par rapport à leurs attitudes et leurs ambitions pour la langue, et aussi dans la façon dont ils perçoivent le rôle linguistique de l'État et de ses institutions quant à la communauté et aux locuteurs ${ }^{17}$. Quant à l'enquête faite exclusivement auprès des locuteurs habituels de l'irlandais dans la région de Múscraí entre 2000 et 2004 , un total de $75,7 \%$ des personnes interrogées pensent que les irlandophones forment une minorité culturelle distincte (101 personnes «d'accord», tandis que 80 des 239 interviewés étaient «fortement d'accord $»)^{18}$.

Cette opinion largement majoritaire parmi les locuteurs de l'irlandais a des conséquences pour la gestion de la langue, mais demeure marginalisée dans la politique nationale en faveur de l'irlandais. Une portion élevée des locuteurs se voit clairement différente de la population nationale en matière d'identité linguistique. Cette identité linguistique centrée sur la langue irlandaise est passée inaperçue dans le discours sur la politique linguistique en Irlande, car il va à l'encontre d'un élément fondamental de l'idéologie de la langue nationale. Des observateurs tels que Reg Hindley ${ }^{19}$ sont allés plus loin en niant qu'il y ait une identité particulière dans la Gaeltacht. Il s'agit d'une mauvaise compréhension de la nature actuelle de l'identité gaélique. Tout le monde peut avoir plusieurs identités en même temps, et ces identités différentes ne sont pas forcement en conflit. Il est certainement vrai que les gens s'identifient à la fois à leur «petit peuple local», leur paroisse, leur «pays», leur comté, leur province et leur nationalité et citoyenneté, mais il y a peu de doute chez les locuteurs quotidiens de la Gaeltacht sur leur situation de minorité linguistique, même s'ils se sentent aussi membres d'une

17. Tadhg Ó HIFEARNÁIN, 2014, op. cit.

18. Tadhg Ó HIFEARNÁIN, «Family language policy, first language Irish speaker attitudes and community-based response to language shift», Journal of Multilingual and Multicultural Development, 2013, 34, p. 348-365.

19. Reg Hindley, The Death of the Irish Language. A Qualified Obituary, 1990, Londres et New York, Routledge, p. 208. 
communauté plus large qui compte aussi des locuteurs-participants plus passifs, comme l'a décrit Nancy Dorian ${ }^{20}$.

Comme la minorité irlandophone de la Gaeltacht existe au sein d'un État qui cherche, au moins officiellement, à promouvoir la langue, les conflits idéologiques subtils mais importants entre le peuple irlandophone et l'État quant à la gestion de la langue irlandaise ne se manifestent pas tous les jours. Cependant, de temps à autre les locuteurs de la Gaeltacht se mobilisent pour demander ce qu'ils considèrent comme étant leurs droits linguistiques. C'est par réponse aux demandes du Gluaiseacht Chearta Sibhialta na Gaeltachta (mouvement des droits civiques de la Gaeltacht), née dans le Connemara à la fin des années 1960, que le gouvernement a créé en 1972 RTÉ Raidió na Gaeltachta, qui diffuse ses émissions des studios de la Gaeltacht au niveau national uniquement en irlandais, et aussi l'autorité locale de développement socio-économique (Údarás na Gaeltachta) en 1979. C'est également grâce à l'appui des locuteurs de la Gaeltacht que la plupart des développements scolaires se font, et que la télévision publique de langue irlandaise, TG4, a vu le jour en 1996.

En fait, on peut constater que l'État gère officieusement la question linguistique depuis les années 1980 comme s'il s'agissait de sauvegarder une petite minorité linguistique d'un coté, mais de promouvoir la langue comme patrimoine culturel ou objet historique pour la population majoritaire, de l'autre. La possibilité de diviser la politique linguistique informellement entre deux objectifs tout à fait différents a créé ses propres problèmes, d'autant plus que le discours officiel du gouvernement reste que l'irlandais est la langue nationale. Acht na dTeangacha Oifigiúla (la loi sur les langues officielles) de 2003 nous fournit un très bon exemple. La loi voulait garantir le droit des citoyens de traiter avec tous les bureaux de l'État et de la fonction publique en irlandais. En décembre 2013, Seán Ó Cuirreáin, le commissaire à la langue, dont le rôle inclut la surveillance de la conformité des organes de l'État à la Loi sur les langues officielles

20. Nancy DORIAN, «Defining the speech community to include its working margins», in Suzanne Romaine (dir.), Sociolinguistic Variation in Speech Communities, 1982, Londres, Edward Arnold, p. 25-33. 
(2003), a démissionné après dix ans de service en signe de protestation contre l'incapacité du gouvernement et de l'appareil étatique de protéger les droits linguistiques de la Gaeltacht et des locuteurs de l'irlandais. En février 2014, une manifestation organisée pour attirer l'attention sur l'échec des politiques linguistiques a rassemblé 10000 manifestants à Dublin, dont des gens de toute la Gaeltacht, soulignant leurs doutes quant à la véracité des objectifs explicites de l'État pour soutenir la langue et ses locuteurs habituels. Ces événements ont illustré le contexte difficile pour la mise en œuvre de nouveaux régimes de planification linguistique dans le Gaeltacht, où l'engagement de l'État est de plus en plus remis en question par les locuteurs habituels, mais aussi par ceux qui s'opposent au statut de l'irlandais.

\section{Les irlandophones hors Gaeltacht et l'avenir de la langue}

Comme nous l'avons vu, les deux tiers des locuteurs habitent en dehors de la Gaeltacht traditionnelle. Liam Mac Mathúna ${ }^{21}$ constate que la plupart des locuteurs de l'irlandais à Dublin passent leur vie sans avoir affaire à la langue traditionnelle de la Gaeltacht. D'après lui, ces gens ne connaissent pas de locuteurs traditionnels, ne vont pas dans l'ouest du pays, n'écoutent pas les émissions de radio et de télévision en provenance de la Gaeltacht et ne voient pas, peut-être, l'intérêt de le faire. On peut démontrer des tendances identiques à Belfast et dans certaines autres grandes villes irlandaises, notamment à l'est du pays. Cependant, il faut se demander si la langue parlée par notre noyau d'environ 80000 locuteurs quotidiens se situe plus près de la langue traditionnelle de la Gaeltacht ou d'une variété linguistique dite nationale, de la revitalisation et de la renaissance, pratiquée dans les milieux des militants linguistiques. Il reste de nombreuses recherches à mener pour comprendre le fait sociolinguistique des locuteurs quotidiens en dehors de la Gaeltacht. Une grande enquête menée auprès de tous les adolescents (15-18 ans) des lycées de la

21. Liam MaC MathúNA, "Linguistic change and standardization», in C. Nic Pháidín, S. Ó Cearnaigh (dir.), A New View of the Irish Language, Dublin, Cois Life, 2008, p. 76-92. 
Gaeltacht de Munster ${ }^{22}$ a montré en fait que les jeunes locuteurs aiment et s'identifient aux variétés non-traditionnelles de l'irlandais que l'on entend dans les écoles bilingues et en ville, tout en disant qu'ils préfèrent la langue traditionnelle de leurs aînés, même s'ils ont du mal à la pratiquer eux-mêmes. Il semblerait qu'il y a bien de la concurrence au niveau du prestige populaire entre l'irlandais traditionnel de la Gaeltacht, le standard national et les variétés parlées des néo-locuteurs ${ }^{23}$. La vie des irlandophones traditionnels nés avant les années 1960, locuteurs qui nous ont donné les atlas linguistiques et les œuvres de dialectologie, la littérature des îles Blasket et les ouvrages des écrivains de Rann na Feirste, les épopées et l'énorme littérature orale, a disparu. De nombreux chercheurs annoncent la mort de la langue avec la fin de cette vie traditionnelle. Pourtant, tous les ans dans les universités nous rencontrons des jeunes locuteurs érudits de la Gaeltacht et d'ailleurs.

Pour l'instant les modèles linguistiques traditionnels sont toujours les variétés-cibles de la formation professionnelle, et il semble aussi que se sont les locuteurs dont la langue est conforme aux usages plutôt traditionnels de prononciation, d'idiome et de grammaire, peu importe leurs origines et même si la langue qu'ils pratiquent n'est pas tout à fait identique au dialecte exact de telle paroisse ou autre, qui sont les mieux placés à parler l'irlandais entre eux et aux enfants futurs. Nous n'avons pas encore fait de recherches pour savoir si cette population de jeunes locuteurs continuera à parler la langue dans le futur, et quelles sont les meilleures politiques linguistiques pour encadrer et soutenir cette minorité de locuteurs actifs au sein de la population nationale et dans un contexte de plus en plus internationalisé.

22. Noel P. Ó MurchadHA, Idé-eolaiocht agus Daonteangeolaiocht na Gaeilge: Aird ar éagsúlacht teanga na Gaeilge [Idéologies et linguistique populaire de l'irlandais : conscience de la variété chez les locuteurs], Thèse de doctorat, Université de Limerick, 2012.

23. Tadhg Ó hIfEARnÁin, Noel P. Ó MurchadHA, «The perception of Standard Irish as a prestige target variety», in T. Kristiansen, N. Coupland (dir.), Standard Languages and Language Standards in a Changing Europe, Oslo, Novus, 2011, p. 97-104. 